\title{
Mechanical Mitral Valve
}

National Cancer Institute

\section{Source}

National Cancer Institute. Mechanical Mitral Valve. NCI Thesaurus. Code C80470.

A valve made of metal, carbon, or artificial materials used to replace the mitral valve in patients with mitral valve stenosis or regurgitation. 